\title{
Database Management System Track for Computer Science Students
}

\author{
Reza Sanati-Mehrizy \\ sanatire@uvsc.edu \\ Computing and Networking \\ Sciences Department
}

\author{
Afsaneh Minaie \\ minaieaf@uvsc.edu \\ Engineering Department
}

\author{
Utah Valley State College \\ Orem, Utah 84058
}

\begin{abstract}
:
Utah Valley State College is a four year college with only undergraduate programs located in an area with many technology oriented business. In our Computer Science program, we offer only one database course titled "Database Theory". Recently we have been examining the content of this database course to make sure that we are covering enough materials in this course. In this process, our database course syllabus has been compared to the course syllabi from several other universities and colleges. This comparison has shown that we basically have been teaching the same subjects as those who have only one database course in their curriculum.
\end{abstract}

Further study and curriculum comparison has shown that several other universities and colleges are offering more than one database course in their computer science program. The reason is that it is impossible to teach all the required subjects (theory, application, administration, etc.) in only one course. Also, experience indicates that the database job market expects our graduate to have enough expertise to be able to install and configure their Database Management System, write application programs, design their database, and maintain and administer their database system. Such an expectation requires the offering of more than one course in this area. Therefore, we have decided that it might be best to add a new area of specialization to our computer science program called the Database Management System track. The paper elaborates the detail content of the curriculum requirement for this Database Management System track in our computer science program.

\section{Introduction:}

Utah Valley State College (UVSC), located in Utah Valley, was founded in 1941. UVSC is a state college comprised of two interdependent divisions. The lower division embraces and preserves the philosophy and mission of a comprehensive community college, while the upper division consists of programs leading to baccalaureate degrees in areas of high community demand and interest ${ }^{1}$. Currently, UVSC offers 13 baccalaureate degrees. UVSC is the fastest growing college in the Utah System of Higher Education with over 23,000 students attending. 
The Bachelor of Science in Computer Science was one of the first Bachelor of Science programs which was approved in 1992 and implemented in $1993^{1}$.

In addition to associate degrees, the Computing and Networking Sciences (CNS) department offers a Bachelor's Degree in Computer Science with four areas of specialization: Computer Science (traditional), Computer Engineering, Software Engineering and Computer Networking. The curriculum content for the Computer Science degree is based on the 2001 ACM Curriculum Report. The CNS department has 10 full time faculties. Half of the faculty have earned a Ph.D. degree and the others have masters degrees. The Computer Science degree at UVSC was accredited by ABET in 2002 and currently has 869 students.

The Bachelor of Science in Computer Science program was one of the first Bachelor of Science programs implemented at UVSC in 1993 . The program's goal has been to provide a quality program that meets accreditation standards while providing students with a skill set that allows them to succeed in computing careers ${ }^{2}$.

In our Computer Science program, we offer only one database course titled "Database Theory". Recently, we have been evaluating the content of our database course to make sure that we are covering enough materials in this course. In this process, our database course syllabus has been compared to the course syllabi from the following Universities and colleges: Brigham Young University, University of Utah, Louisiana State University in Shreveport, Utah State University, and Union Community College in Logan, Utah. This comparison has shown that that we basically have been teaching the same subjects as those who have only one database course in their curriculum.

Further study and curriculum comparison showed that some other universities are offering more than one database course. The reason is that it is impossible to teach all the required subjects (theory, application, administration, etc.) in only one course. Also, experience indicates that the database job market expects our graduates to have enough expertise to be able to install and configure their Database Management System, write application programs, design the database, maintain and administer their database system. Therefore, we decided that it might be best to add a new area of specialization to our Computer Science program called Database Area of Specialization.

One of our advisors asked two students who changed their majors from an AS degree to BS degree in computer science what classes helped them or what classes they were willing to take and both mentioned database classes. Both of them gained from their experience and believe they accomplished the objective of the course.

The advisor also did an exit interview with several students who completed the Internship class (CNS 481R). They were asked what classes helped them, and what classes they were encouraged to take based on the internship. By far, the most common subject was the database classes. They appreciated what has been taught in CNS 3520 (Database Theory) and wanted to take more classes in this area.

\section{Database Area of Specialization}


Our study and curriculum comparison indicates that there is (at least in our area) a great need for covering more materials than what we are currently covering in our database class. Since we currently offer only one course in database area, only a limited amount of materials can be covered in class. Unfortunately, we can not add more courses in this area to our curriculum because this requires eliminating some existing courses which are required by ABET or due to other reasons. Therefore, we have concluded that the best option for us is to have a new database track in our Computer Science program.

Since the department of Computer Information Technology and Education (CITE) at UVSC offers some applied courses in database area, CNS faculty have been discussing this issue with CITE in joint meetings. After reviewing industrial demands, our goals for database coverage, and the courses offered in CITE, a possible curriculum for our database track can be proposed as the following:

In order to graduate with a Computer Science degree, students must complete 123 semester hours of course work. These 123 credit hours include 36 credit hours general study, 45 credit hours of Computer Science core requirements, and 42 credit hours requirements for student graduating in database area of specialization listed as follows.

\section{General Study (36 Credit Hours)}

All students graduating from UVSC must complete the following 36 credit hours general study.

- ENGL 1010

- ENGL 2020

- PHIL 2050

- ECON or HIST or PLSC

- HLTH 1100 or PES 1300

- COMM 1100

- COMM 2010

- PHYS 2210 \& $221 \mathrm{~L}$

- PHYS 2220 \& 222L

- FINE ART

- Biology

\section{0}

3.0

3.0

3.0

2.0

3.0

3.0

5.0

5.0

3.0

3.0

\section{Computer Science Core Requirements (45 Credit Hours)}

- CNS 1250

- CNS 1350

- CNS 1380

- CNS 1510

- CNS 2300

- CNS 2400
Object-Oriented Programming I

3.0

Object-Oriented Programming II

Assembly Language \& Computer Architecture

3.0

Unix Operating Systems

3.0

Discrete Structure I

Object-Oriented Data Structures
3.0

3.0

3.0 
- CNS 2600 Fundamentals of Data Communications 3.0

- CNS 301R Invited Speakers Series 1.0

- CNS 3060 Operating Systems Theory 3.0

- CNS 3240 Introduction to Computational Theory 3.0

- CNS 3690 Advanced Topics in Data Communications 3.0

- Math 1210 Calculus I 5.0

- Math 1220 Calculus II 5.0

- Math 2230 Principles of Statistics I \& II 4.0

\section{Database Area of Specialization Requirements (42 Credit Hours)}

All students graduating in database area of specialization must complete 42 credit hours which consist of 27 credit hours core and 15 credit hours electives:

\section{Database Core Courses:}

\section{One of the followings:}

CNS 3220, Visual Basic Software Development

CNS 3250, Java Software Development

CNS 3260, C\#.net Software Development

CNS 3370, Advanced C++ Software Development

2. MCT 2240, Interaction Design I (3.0 CR)

Uses a systems approach in designing interactive multimedia products to create user experiences that enhance and extend the way people work and communicate. Stresses an iterative process of design and evaluation based on theory and good practice are needed to create usable products.

Prerequisite(s): MCT 1210

\section{CNS 2520, Database Theory (3.0 CR)}

Introduces theory, concepts, architecture, and use of Database Management Systems (DBMS). Presents the relational and object-oriented database models used in both centralized and client/server databases. Discusses basic constraints and Structured Query Language (SQL), database models, History of database systems, database design, entity relationship and enhanced entity relationship, UML, primary key, foreign key, mapping ER/EER to schema, functional dependencies and normalization process, merging schemas, indexing, disk storage, basic file structures and hashing, relational algebra and relational calculus relating to database management system, join operations (inner join, outer join, semi join), etc.

Prerequisite: CNS 2400.

\section{CITE 3410, Database Systems (3.0 CR)}

Advanced SQL queries, DB Programming (Stored Procedures, Triggers, Control Structures), Physical design/physical environment (Data types, Physical Integrity, DBMS managed constraints, Primary key, Foreign key, Databases in 
Internet/Client Server environments, Front end/Back end connectivity (ADO.NET, OBDC, OLE DB, JBDC), Connections, Commands, Data sets, Introduction to Database Administration (Concurrency issues, Transaction Processing, Backup and recovery), Introduction to Data warehousing, Introduction to Distributed databases.

Prerequisite: CNS 2520 (Database Theory) or CITE 2950 (Database Fundamentals).

\section{CNS 3400, Software Engineering I (3.0 CR)}

Presents concepts, methodology and best-practices necessary to develop large scale software projects. Includes step-wise software requirements analysis, design, implementation, testing and release. Discusses software generation, reuse, scheduling, verification, and maintenance. Emphasizes current "real world" industry best-practices and tools.

Prerequisite: CNS 2400.

\section{CNS 4100, Database Management System Construction (3.0 CR)}

This course looks at issues involved in actually implementing a DBMS.

Students will implement from scratch a relational DBMS with added features such as indexing.

Prerequisite: CITE 3410 and 3220 or 3250 or 3260 or 3370 .

\section{CNS 4190, Enterprise Architecture (3.0 CR)}

Development of enterprise systems using distributed software architecture and frameworks. Explores leading distributed systems standards for platforms, languages, and data independence. Focuses on server-side development taking into consideration componentization, transaction management, persistence, and security. Uses Java enterprise servers, the J2EE architecture, server pages, the Common Object Request Broker Architecture (CORBA), and XML standard to develop enterprise-level distributed object systems capable of secure Internet transaction processing. This course will be updated to cover Web Services/.NET. Prerequisite: CITE 3410 and CNS 3690 and 3220 or 3250 or 3260 or 3370.

\section{CITE 4200, Database Administration (3.0 CR)}

Installation and configuration, Setting up the operational environment, Setting up users and permissions, securing the database, distributed databases, clustering, replication, Performance monitoring and tuning, reconfiguring the database, developers vs. operational needs vs. management needs, Backup and recovery. Prerequisite: CITE 3410.

\section{CNS 4500, Advanced topics in Database Systems (3.0 CR)}

Transaction Processing, Concurrency Control Techniques, Database Recovery Techniques, Database Security and Authorization, Database Integrity, Distributed Databases and Client-Server Architectures, Load balancing. Data Warehousing, Data Mining, Database Machines, Mobile Database, Multimedia Database, GIS, 
Genome Data Management, Data Fragmentation, Data Encryption, Locking, Deadlock, etc.

Prerequisite: CITE 3410.

\section{Database Area of Specialization Electives (15 Credit Hours)}

Students graduating in database area of specialization must complete an additional 15 credit hours from the following list of electives:

- CNS 4470 Artificial Intelligence 3.0

- CNS 3660 Web Server Administration Programming $\quad 3.0$

- CNS 3670 Network Programming 3.0

- CNS 4410 Human Factors in Software Engineering 3.0

- CNS 3220 Visual Basic Software Development or

CNS 3250 Java Software Development or CNS 3260 C\#.net Software Development or CNS 3370 Advanced C++ Software Development $\quad 3.0$

- $\quad$ CNS 481R Internship Work Experience (max of 3) 3.0

- CNS 3400 Software Engineering I 3.0

- CNS 4400 Software Engineering II 3.0

- CNS 4230 Software Testing and Quality Assurance 3.0

- CNS 3550 Internet Software Development 3.0

- CNS 4510 Operating System Design and Simulation $\quad 3.0$

- CNS 3540 Game Programming 3.0

More courses from the list of database electives can be transferred to the list of database core courses if someone desires to do so.

\section{Conclusion}

Students graduating in computer science at UVSC in the database area of specialization will have a strong background in this field which makes them more marketable. Graduating students with this extensive database knowledge satisfies the area's industrial demand. Also, having such an area of specialization will have positive effects on our program enrolment.

Other programs may select any of these new database courses as core or elective to be included in their curriculum. Higher education institutions abroad may benefit from implementing this area of specialization in their computer science program.

\section{References}

[1] http://www.uvsc.edu , UVSC - Department of Computing and Networking Sciences, access on December 2004. 
[2] Computer Science Program Self Study for the 2002-2003 ABET Accreditation Cycle, Computing and Networking Sciences Department, Utah Valley State College, 2002.

\section{Biography}

REZA SANATI MEHRIZY is an associate professor of the Computing and Networking Sciences Dept. at Utah Valley State College, Orem, Utah. He received his MS and $\mathrm{PhD}$ in Computer Science from University of Oklahoma, Norman, Oklahoma. His research focuses on diverse areas such as: Database Design, Data Structures, Artificial Intelligence, Robotics, and Computer Integrated Manufacturing.

AFSANEH MINAIE is an associate professor in the Engineering Department at Utah Valley State College. She received a B.S., M.S. and Ph.D. all in Electrical Engineering from University of Oklahoma in 1981, 1984 and 1989 respectively. Her current interests are in computer architecture, embedded systems, digital design, and computer interfacing. 